\title{
Interface state effects in GaN Schottky diodes
}

\author{
A. Ahaitouf ${ }^{a, b, *}$, H. Srour ${ }^{c, d}$, S. Ould Saad Hamady ${ }^{c, d}$, N. Fressengeas ${ }^{c, d}$, A. Ougazzaden ${ }^{a, e}$, J.P. Salvestrini ${ }^{c, d}$
}

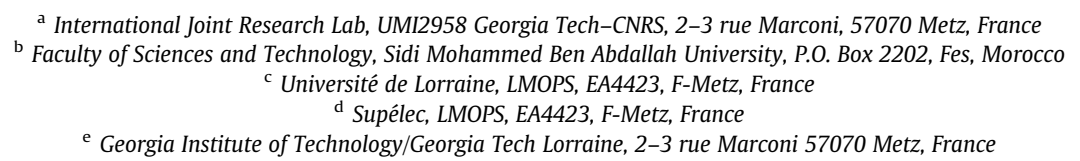

Current voltage (I-V) and capacitance voltage $(\mathrm{C}-\mathrm{V})$ measurements have been performed versus temperature on GaN Schottky diodes. The results show an increase of the Schottky barrier height $\phi_{b}$ and a decrease of the ideality factor $n$ both with the increase of the temperature. We show that this behavior originates in the existence of an interface state density distribution, which is determined via the analysis of the temperature dependence of the I-V measurements, and allows the tunneling of the carriers from the semiconductor to the metal. Those interface states are shown to be responsible for interface inhomogeneities which result in two Gaussian voltage dependent Schottky barrier distributions. We show also that, in the presence of this interface state distribution, C-V measurements, without the correction of the built in voltage by taking into account the effect of both the high values of the ideality factor and series resistance, lead to erroneous values of the Schottky barrier height $\phi_{b}$.

\section{Introduction}

Group III nitride wide band gap semiconductors have attracted considerable interest owing to their applications for optical devices operating in the blue and ultraviolet wavelength regions. These materials and especially gallium nitride ( $\mathrm{GaN}$ ), were also found to be suitable for operation at high electrical and optical power levels, high temperatures and in harsh environments [1-8]. Schottky contact on n type GaN, which is critical for the realization of all these electronic and optoelectronic devices, has been extensively studied $[9,2,3,5,10,11,6,12-17]$. The ideality factor $n$ and more especially the barrier height $\phi_{b}$ are the most important characteristics of metalsemiconductor Schottky barrier contacts, which should be optimized to achieve high performance devices. As seen in Table 1, several values of the Schottky barrier heights and ideality factors have been reported in the literature, depending on the metal used (Pt, Au, Ni and Pd) for the Schottky contact and the active layer growth technique and conditions. The values of the Schottky barrier height (SBH) $\phi_{b}$ and ideality factor $n$ were found to range between $0.51 \mathrm{eV}$ and $1.13 \mathrm{eV}$ and 1.03 and 2.96, respectively. This large spread of the values, except those of Schmitz et al. [10] who have obtained values of the ideality factor that are independent of the metal type used, indicates that both the metal

\footnotetext{
* Corresponding author at: Faculty of Sciences and Technology, Sidi Mohammed Ben Abdallah University, P.O. Box 2202, Fes, Morocco. Tel.: + 212 665202700; fax: + 21235 5608214.

E-mail address: ali.ahaitouf@georgiatech-metz.fr (A. Ahaitouf).
}

type of the Schottky contact and the technique used for the growth of the active layer, and thus the interface between them, play an important role for the achievement of a high quality Schottky diode. It is to be noticed that Schottky contacts realized with Pt are the most often used in the literature. Many authors $[12,16,18,19]$ have also reported an increase of the value of $\phi_{b}$ with increasing temperature. This unexpected temperature dependence was attributed to some inhomogeneities of the Schottky barrier $[18,19]$ or to the tunneling effect in a highly defective material [12].

All these results show that in spite of the numerous studies for the realization of electrical contacts on GaN materials, the debate concerning the conduction mechanisms which govern the current flow in the nitride GaN based structures is still open. Additional works are thus suitable for a better control of this kind of contact and for a unified interpretation of the conduction phenomenon.

In this work, $\mathrm{Pt} / \mathrm{n}-\mathrm{GaN}$ Schottky diodes have been realized and current-voltage (I-V) as well as capacitance-voltage (C-V) measurements versus temperature have been performed and analyzed. The results show that the structures under test present an important interface states distribution which is temperature dependent. These interface states act as centers favoring the tunneling of the carriers in a more important way than the tunneling through the top of the barrier. Their overall effect can be seen as the effect of an interfacial layer explaining the temperature dependence of both the barrier height $\phi_{b}$ and the ideality factor $n$. At last, values of the barrier height $\phi_{b}$ derived from I-V and C-V measurements, respectively, are shown to be consistent if, in the case of $\mathrm{C}-\mathrm{V}$ measurements, the value of the built in voltage is corrected to take into account the interface state effect. 
Table 1

Values of the Schottky barrier and ideality factor in GaN, obtained by different authors using different metals and growth techniques of the active layer (LPMOCVD [10], MOVPE [2,14], MOCVD [3,16,18,20], MBE [11]).

\begin{tabular}{lll}
\hline Metal type & $\begin{array}{l}\text { Barrier height } \\
\phi_{b}(\mathrm{eV})\end{array}$ & $\begin{array}{l}\text { Ideality factor } \\
n\end{array}$ \\
\hline $\mathrm{Pt}$ & $1.03-1.04[2]$ & $1.21[2]$ \\
& $0.52-1.13[3]$ & $1.05-2.96[3]$ \\
& $1.08[10], 0.88[14]$ & $1.04[10], 1.1[14]$ \\
& $0.99[18]$ & $1.42[18]$ \\
$\mathrm{Au}$ & $0.88[3,10]$ & $1.06[3], 1.04[10]$ \\
& $0.82[16]$ & $1.15[16]$ \\
$\mathrm{Ni}$ & $0.844[9], 1.1[11]$ & $1.03[9], 1.4[11]$ \\
& $0.99[10], 0.61-0.69[12]$ & $1.04[10], 1.19[12]$ \\
$\mathrm{Pd}$ & $0.75[18], 0.82[20]$ & $1.98[20]$ \\
& $0.91-0.94[2], 1.05-1.11[3]$ & $1.14[2], 1.02-1.2[3]$ \\
& $0.92[10], 0.69[18]$ & $1.04[10], 1.58[18]$ \\
& $0.51-0.97[21]$ & $2[21]$ \\
\hline
\end{tabular}

\section{Experimental procedures}

\subsection{Samples preparation}

The samples used in this study are $3.5 \mu$ m thick n-GaN layers grown by metalorganic vapor phase epitaxy (MOVPE) on sapphire substrates supplied by LUMILOG with a nominal doping concentration ranging between $1 \times 10^{18} \mathrm{~cm}^{-3}$ and $3 \times 10^{18} \mathrm{~cm}^{-3}$. Before metallization, the samples were cleaned in acetone and etched in $\mathrm{HCl}(3 \mathrm{~mol} / \mathrm{l})$ solution for $5 \mathrm{~min}$ to remove the native oxide. The Schottky diodes were fabricated on the cleaned surface using photolithography technique. For ohmic contact, Ti/Al/Ti/Au (15/200/15/200 nm) layers were deposited by thermal evaporation and then annealed at $500{ }^{\circ} \mathrm{C}$ for $15 \mathrm{~min}$ in a flowing $\mathrm{N}_{2}$ atmosphere. A $150 \mathrm{~nm}$ Pt thick layer was deposited (using an e-beam evaporation system) to form a circular Schottky contact with a surface equal to $7.07 \times 10^{-4} \mathrm{~cm}^{2}$. I-V and $\mathrm{C}-\mathrm{V}$ measurements were performed versus temperature from $77 \mathrm{~K}$ to $300 \mathrm{~K}$, using a Keithley Source Measure Unit 2602 and a HP 4192 impedance analyzer, respectively.

\subsection{I-V measurements}

Fig. 1 shows the voltage dependence of the current density $J$ obtained in a Pt/n-GaN Schottky diode at different temperatures. For relatively large voltage $(>0.7 \mathrm{~V})$ the current saturates due to effect of the series resistance [22]. For lower voltage, the characteristic exhibits two regimes characterized by two different slopes corresponding to different transport mechanisms. The one taking place in the intermediate voltage range $(0.2-0.6 \mathrm{~V})$ was shown [5] to be driven by the thermionic emission (TE) and is preponderant at high temperatures. By contrast, in the low voltage range $(0-0.45 \mathrm{~V})$, the current flow is dominated by the thermionic field emission (TFE). This is especially the case for highly doped materials.

The TE and TFE transport mechanisms can be described respectively [22,23] by:

$$
\begin{aligned}
J_{T E}= & A^{*} T^{2} \times \exp \left(-\frac{q \phi_{b}}{k T}\right) \times \exp \left(\frac{q\left(V-R_{s} I\right)}{n k T}\right) \\
& \times\left[1-\exp \left(\frac{-q\left(V-R_{s} I\right)}{k T}\right)\right]
\end{aligned}
$$

and

$$
\begin{aligned}
J_{T F E}= & A^{*} T \frac{\sqrt{\pi E_{00} q\left(\phi_{b}-V-\xi\right)}}{k \cosh \left(E_{00} / k T\right)} \times \exp \left[\frac{q\left(V-R_{s} I\right)}{E_{00} \operatorname{coth}\left(E_{00} / k T\right)}\right] \\
& \times \exp \left[-\frac{q \xi}{k T}-\frac{q\left(\phi_{b}-\xi\right)}{E_{00} \operatorname{coth}\left(E_{00} / k T\right)}\right] .
\end{aligned}
$$

The total current is then the sum of both. $A^{*}$ is the Richardson constant equal to $26.4 \mathrm{Acm}^{-2} \mathrm{~K}^{-2}$ for n-type GaN [16], $k$ is the

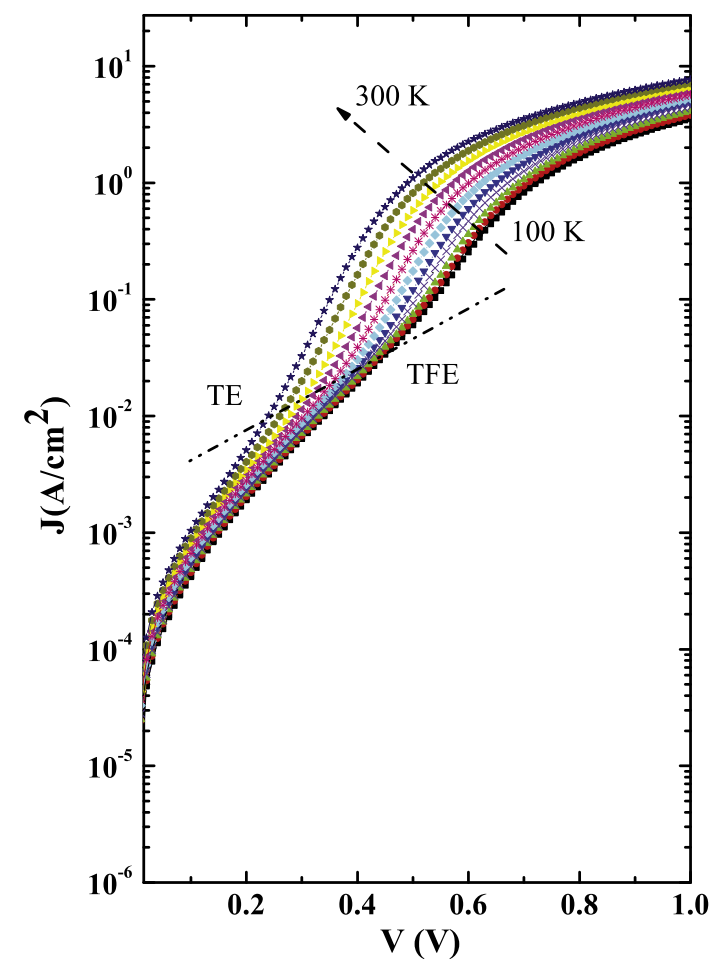

Fig. 1. Temperature dependence of the $J-V$ characteristics in a Pt/n-GaN Schottky diode. Temperature increases by step of $20 \mathrm{~K}$ in the arrow direction. The dot-line indicates the separation between the two regimes: behind this line the TFE is preponderant, above this line the TE takes place.

Boltzmann constant, $T$ is the absolute temperature, $q$ is the electronic charge, $\phi_{b}$ is the effective Schottky barrier height, $n$ is the ideality factor, $E_{00}$ is the tunneling factor, $\xi$ is the energy of the Fermi level of the semiconductor with respect to the bottom of its conduction band, $R_{S}$ is the series resistance and $V$ is the applied bias voltage.

\subsection{C-V measurements}

Fig. 2 shows an example of $\mathrm{C}-\mathrm{V}$ and $1 / C^{2}-V$ curves obtained in a $\mathrm{Pt} / \mathrm{n}-\mathrm{GaN}$ Schottky diode at different temperatures and a frequency of $500 \mathrm{kHz}$ using a parallel RC model. The data of $1 / C^{2}-V$ in Fig. 2 show a linear behavior of the $1 / C^{2}$ plot versus $V$, indicating a uniform doping concentration profile whatever the temperature.

This doping concentration can be obtained from the slope of the $1 / C^{2}-V$ plot by the following relationship, where $\epsilon_{0}$ and $\epsilon_{S C}$ are the vacuum dielectric permittivity and the semiconductor relative dielectric permittivity respectively:

$N_{D}=-\frac{2}{q \epsilon_{0} \epsilon_{S C}} \times \frac{d V}{d\left(S^{2} / C^{2}\right)}$

The value of the doping density, at room temperature, was found equal to $5 \times 10^{18} \mathrm{~cm}^{-3}$. However, this value must be corrected [24,25] to take into account the higher-than-one value of $n$, as we will see in the next section. After correction using the mean values of $n$ (see next section), the values of $N_{D}$ becomes equal to $3.9 \times 10^{18} \mathrm{~cm}^{-3}$.

\section{Series resistance and ideality factor}

The series resistance $R_{S}$ is an important parameter in the characterization of Schottky barrier diodes because it limits the conduction process particularly in large band gap semiconductor materials such as GaN. This parameter has been determined using the Cheung and Cheung [26] technique. The obtained values of $R_{s}$ are then used to 


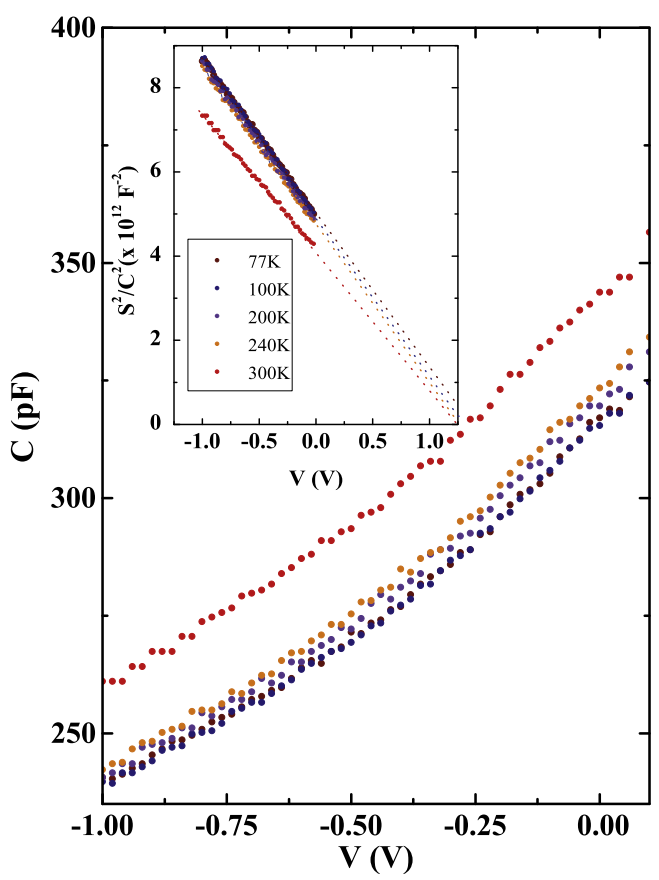

Fig. 2. $\mathrm{C}-\mathrm{V}$ (measured at a frequency of $1 \mathrm{MHz}$ ) and $1 / \mathrm{C}^{2}-V$ dependencies in $\mathrm{Pt} / \mathrm{n}-\mathrm{GaN}$ Schottky diode structures obtained at different temperatures.

correct the I-V characteristics and finally obtain the ideality factor $n$ by a fitting procedure based on a least square method using Eqs. (1) and (2). The temperature dependence of the derived values of $R_{S}$ and $n$ are reported in Fig. 3 .

The series resistance $R_{s}$ decreases with increasing temperature, and reaches, at room temperature, a value equal to 90 which is a rather large value. Fig. 3 also shows that the values of $n$ range between 1.9

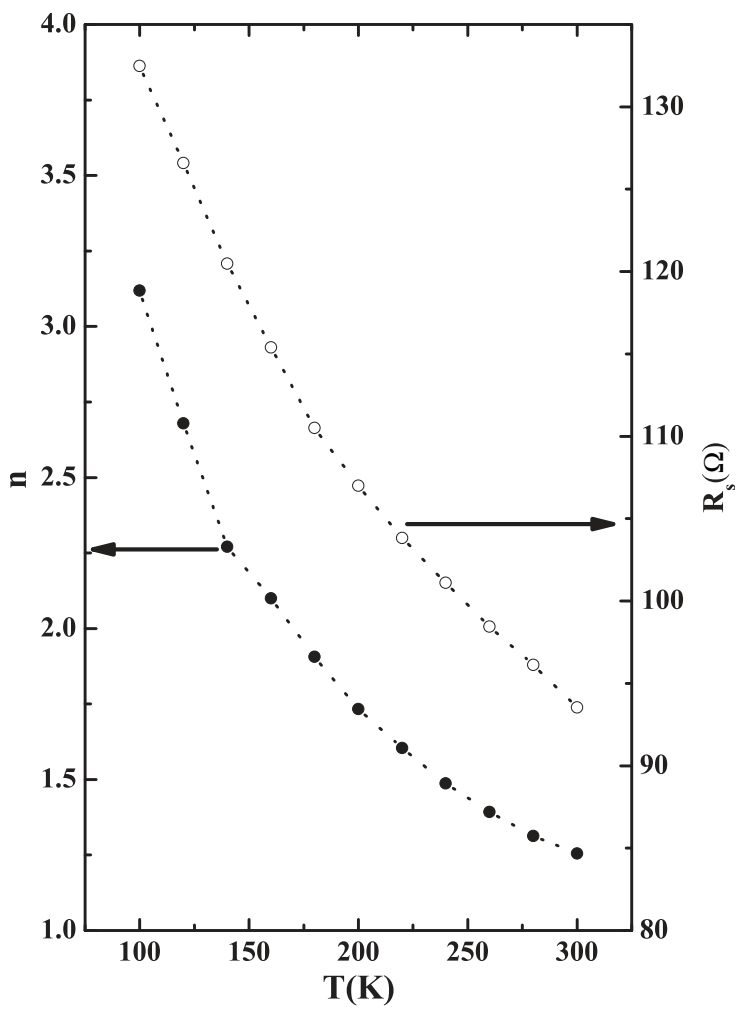

Fig. 3. Temperature dependence of the ideality factor and series resistance in Pt/n-GaN Schottky structures. and 1.3 in the temperature range $180-300 \mathrm{~K}$, and become larger than 2 for lower temperatures. This will be discussed in terms of inhomogeneous SBH in Section 5. The same dependence of $n$ on temperature has been reported for ( $\mathrm{Au}, \mathrm{Pt}, \mathrm{Pd}, \mathrm{Ni}) / \mathrm{n}-\mathrm{GaN}$ Schottky diodes $[16,18]$, with values of $n$ in rather good agreement with our results. This decrease of $n$ with temperature has been interpreted in several ways. It has been described as the $T_{0}$ effect (according to which the ideality factor follows the empirical relation $n=1+\frac{T_{o}}{T}$ owing to a tunneling, or a particular distribution of interface states or a non-uniformly doped surface layer and even a combination of these [22]), and attributed to fluctuations of the barrier height by Werner and Güttler [27] and Tung [28], as well as to the presence of a high interface state density by Levine [29] and Crowell [30]. The value of $n$ obtained at room temperature $(n=1.3)$ is in good agreement with the values reported in the literature $[5,10,14,17,18]$ for $\mathrm{Pt} / \mathrm{n}$-GaN Schottky diodes. Its higher-than-unity value can also be attributed to a high doping concentration $\left(3.9 \times 10^{18} \mathrm{~cm}^{-3}\right)$, since it is well known that the ideality factor departs substantially from unity when doping is increased [23].

\section{Zero bias Schottky barrier height}

The value of the Schottky barrier height has been deduced from the data of Fig. 1 according to Eq. (1) by:

$\phi_{b}=-\frac{k T}{q} \ln \frac{J_{s}}{A^{*} T^{2}}$,

where $J_{s}$ is the saturation current density obtained by extrapolating the logarithmic I-V plot to $V=0$. As shown in Fig. 4-a, the value of $\phi_{b}$ increases linearly with increasing temperature, opposite to what could be expected for an ideal diode: the barrier height should decrease with temperature, owing to the lattice expansion [22]. This increase of $\phi_{b}$ has also been reported for (Au, Pt, Pd, Ni)/n-GaN Schottky diodes $[16,18]$. The TFE mechanism, which is a tunneling through the top of the barrier, can induce barrier height lowering and ideality factor increase with temperature.

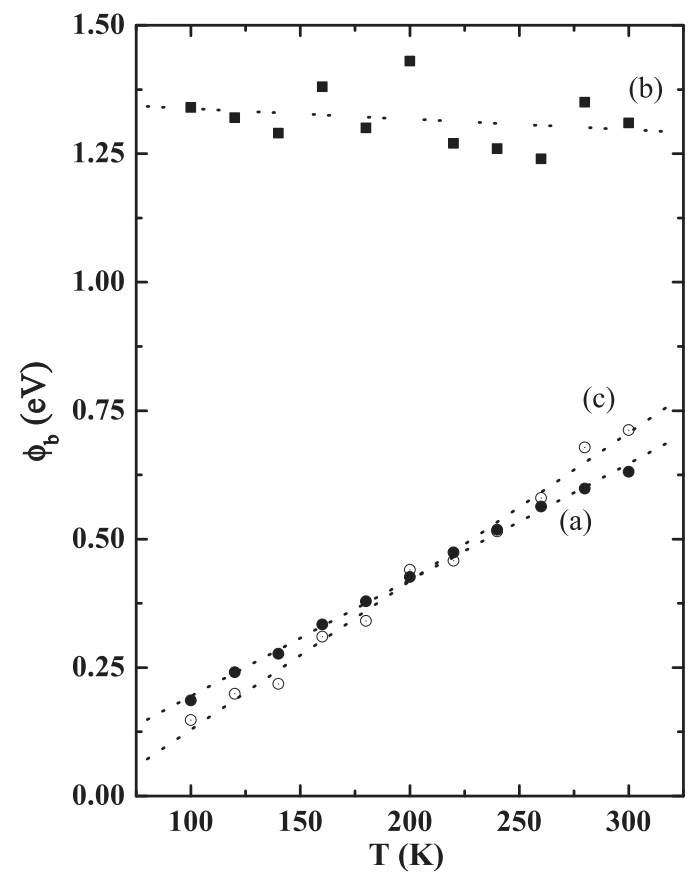

Fig. 4. Temperature dependence of the effective barrier in $\mathrm{Pt} / \mathrm{n}-\mathrm{GaN}$ Schottky diodes as deduced from a) I-V measurements ; b) C-V measurements ; c) C-V measurements after correction. 
The effective barrier and ideality factor variations due to this tunneling effect are given respectively by $[27,22]$ :

$\Delta \Phi_{\text {tunn }}(T)=\left(1.5 \times E_{o o}\right)^{\frac{2}{3}} \times V_{b i}^{\frac{1}{3}}(T)$

and

$n_{\text {tunn }}(T)=\frac{q E_{o o}}{k T} \operatorname{coth}\left(\frac{q E_{o o}}{k T}\right)$

where $V_{b i}$ is the built-in voltage as deduced from the intercept of the $1 / C^{2}-V$ plot (see Fig. 2 ), $\hbar$ is the reduced Planck constant and $E_{o o}$ is the tunneling energy given by:

$E_{o o}=\frac{\hbar}{2} \sqrt{\frac{N_{D}}{m^{*} \epsilon_{o} \epsilon_{s c}}}$

Using the values of $V_{b i}(\mathrm{~T})$, the barrier height variation has been estimated and plotted in Fig. 5.

It shows that $\Delta \Phi_{\text {tunn }}$ increases linearly with temperature, and that the contribution of this tunnel effect to the barrier height variation is roughly equal to ten $\mathrm{meV}$ in the considered temperature range, indicating a decrease of the SBH with increasing temperature. Thus the TFE effect cannot be responsible for the variations of $\phi_{b}$ shown in Fig. 4-a. This is reenforced by the calculated temperature dependence, according to Eq. (6), of the ideality factor which is plotted in Fig. 5. It results in a linear increase of the value of $n_{\text {tunn }}$ from 1.1 to 1.3 for temperatures varying between $77 \mathrm{~K}$ and $300 \mathrm{~K}$, in contrast to our experiment which shows a decrease of the ideality factor (see Fig. 3) with the increase of the temperature.

Here we assume that this unexpected temperature dependence of $\phi_{b}$ is due to an unintentional interfacial layer, which can appear at the surface of the sample during the fabrication process or can be due to an interface state effect linked to structural defects in the active layer such as nitrogen anti-site or vacancies, for example. Card

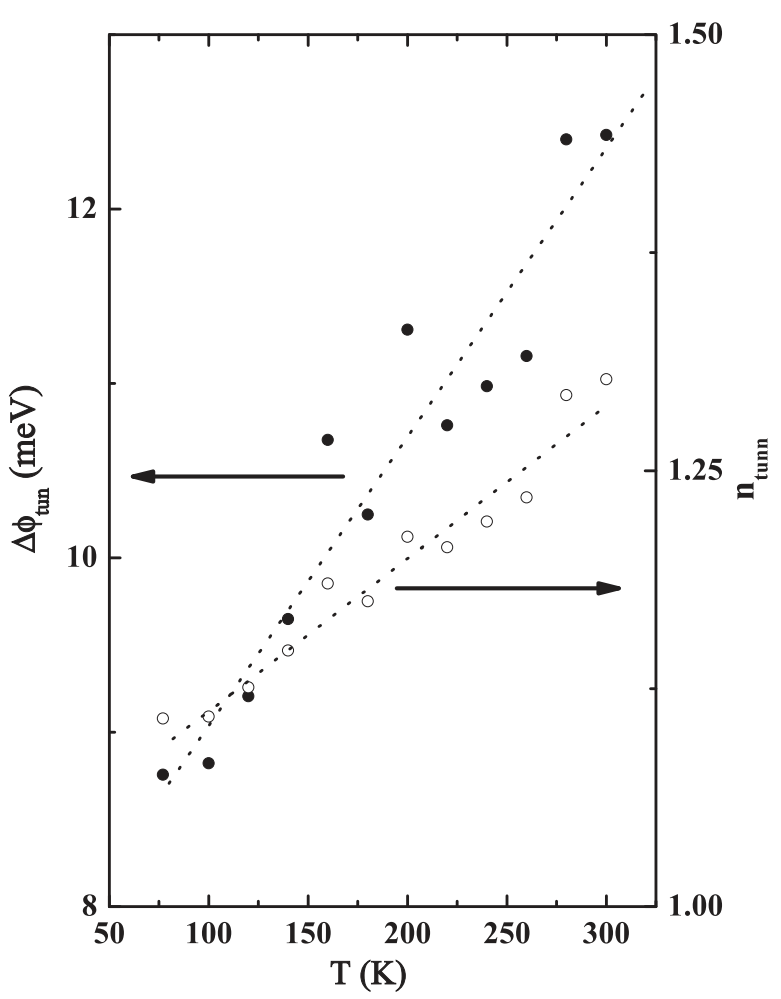

Fig. 5. Temperature dependence of the TFE contribution to the Schottky barrier height and ideality factor variations for the studied structures. and Rhoderick [31] have reported, in the case of the presence of a thin interfacial layer between the metal and the semiconductor, that the zero bias barrier height follows a linear variation versus temperature given by:

$\phi_{b}(T)=\phi_{b 0}^{0 K}+(\beta-\alpha) T$

where $\phi_{b 0}^{O K}$ is the zero absolute barrier height, $\beta$ is the tunnel coefficient depending on the thickness and the barrier height of the interfacial layer and $\alpha$ is the temperature band gap variation. Eq. (8) applied to the data of Fig. 4-a gives a slope of $\beta-\alpha=1.95 \times 10^{-3} \mathrm{eV} / \mathrm{K}$ and a $\phi_{b 0}^{0 K}$ of $0.16 \mathrm{eV}$. Thus we suggest the existence of an additional tunneling effect via the interface states, acting as an interfacial layer, as being responsible for these behavior of $\phi_{b}$ and $n$ versus temperature.

\section{Interface state effects}

The changes in the slope of the $J-V$ curves observed in Fig. 1 reveal a change in the ideality factor $n$ as a function of the voltage. These variations, as deduced from Eq. (1), and given by:

$n=\frac{q}{k T} \times \frac{d V^{\prime}}{d\left[\log \left(\frac{J_{T E}}{1-\exp \left(-\frac{q^{\prime}}{k T}\right)}\right)\right]}$

are shown in Fig. 6. $V^{\prime}=V-R_{s} I$ is the effective applied voltage. For each temperature, $\mathrm{n}$ increases with the increase of the voltage, up to a maximum and then decreases. Such behavior has been reported elsewhere on oxidized surfaces of GaAs [32] and InP [33] and has been attributed to interface state effects. It is to be noted that the peak in the $n(V)$ curve (Fig. 6) can be fully hidden, as it is the case for instance in ref [18], if in Eq. (9) the series resistance effect is not taken into account [25].

When these interface states are in equilibrium with the semiconductor, the interface state density is linked to the ideality factor by [22]:

$N_{s S}=\frac{\epsilon_{o} \epsilon_{i}}{\delta q^{2}}(n-1)-\frac{\epsilon_{o} \epsilon_{S C}}{q^{2} W}$,

where $W$ is the depletion width, $\epsilon_{i}$ and $\delta$ are respectively the relative dielectric constant and the thickness of the interfacial layer. The

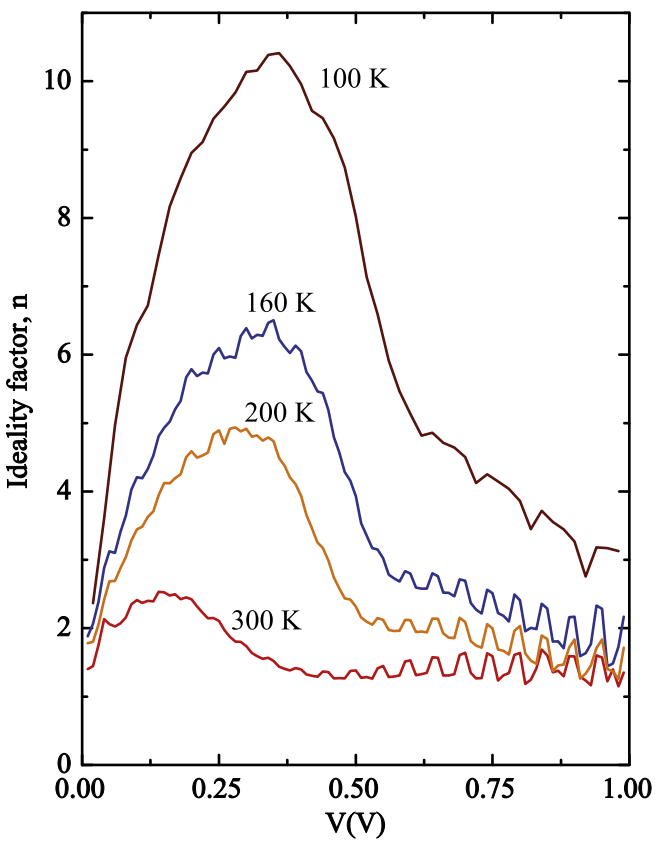

Fig. 6. Voltage dependence of the ideality factor $n$ in Pt/n-GaN structures. 
thickness of this layer was found to be larger than $3 \mathrm{~nm} \mathrm{[34]} \mathrm{in} \mathrm{air}$ exposed GaN layer. Using Eq. (10) and data of Fig. 6, the voltage dependence $\mathrm{N}_{s s}(\mathrm{~V})$ can be determined. According to the relationship $E_{c}-E=\phi_{b 0}-q V^{\prime}$ [18], where $\phi_{b 0}$ is the room temperature value of the barrier height, the energy dependence of the interface state distribution $\mathrm{N}_{s s}\left(E_{c}-\mathrm{E}\right)$ has been derived and plotted on Fig. 7 assuming a $4 \mathrm{~nm}$ thick layer. This approach has been used in the case of GaAs [32] and InP [33], showing results in good agreement with those obtained via deep level transient spectroscopy (DLTS) measurements. This technique has the advantage of giving a rapid way to get a correct estimation of the state density involved in the conduction mechanism since, these states being in equilibrium with the semiconductor, they allow charge transfer to, or from, the conduction band by capture and emission mechanism.

As shown in Fig. 7, the energy dependence of $\mathrm{N}_{s s}$ exhibits the same behavior whatever the temperature. The interface state density shows a broad peak centered at an energy which depends on the temperature. These energy values, ranging between $0.35 \mathrm{eV}$ and $0.55 \mathrm{eV}$ according to the temperature, are in good agreement with those obtained by Cho et al. [35] (0.5-0.6 eV), Gotz et al. [36] $(0.49 \mathrm{eV})$, and Tsarova et al. [37] (0.6 eV) using DLTS in GaN Schottky diodes, and are attributed to nitrogen anti-sites. These peaks can also originate from an unintentional surface oxidization which can occur during the fabrication process steps. Additional work is needed to precisely determine its origin.

The interface states play a major role in the charge transfer to or from the conduction band by capture and emission mechanisms or with the metal by tunneling. These states behave like an interfacial layer through which carriers can move to the metal by tunneling and are responsible for the large variations of $\phi_{b}$ and $n$ versus temperature. As shown from the temperature dependence of the interface states density (see inset of Fig. 7), a temperature decrease leads the Fermi level to move into a higher interface state density, which makes it difficult to move, and consequently partially or completely pins the surface potential variations under an applied voltage. This results in an apparent higher ideality factor (see Fig. 6). The interface states assist the tunneling of carriers to the metal and result in a higher current flow through the structure, as a consequence the

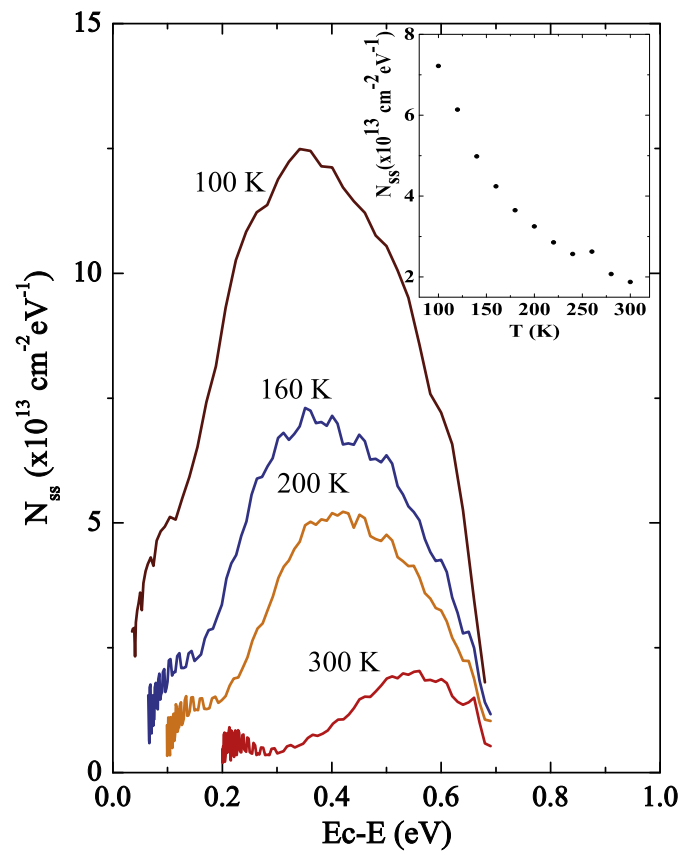

Fig. 7. Interface state density distribution below the conduction band edge in the studied structures. The inset shows the temperature dependence of $N_{s s}$ at $E_{c}-E=0.6 \mathrm{eV}$, as deduced from the data of the main figure. apparent Schottky barrier height is reduced. By contrast, when temperature increases, the interface states are partially or completely filled, making the surface potential variations easier, and the corresponding ideality factor smaller. The current then follows the thermionic emission behavior and the measured barrier height is thus closer to the theoretical expected values.

These interface defects can also be responsible for some inhomogeneities of the Schottky barrier height as reported by Mamor et al. [18] and Mtangi et al. [19]. To confirm this interpretation, according to the data of Figs. 3 and 4-a, we have plotted both the variations of $\phi_{b}$ and $\left(n^{-1}-1\right)$ versus $\frac{1}{2 k T / q}$ (see Fig. 8 ). Both quantities exhibit linear dependencies with two different slopes corresponding to two different temperature ranges with a transition at 180. According to the model of the barrier inhomogeneities at Schottky contacts [27], the SBH is spatially distributed and can be described by a Gaussian distribution as:

$\phi_{b}=\bar{\phi}_{b}-\frac{q}{2 k T} \sigma_{s}^{2}$

where $\phi_{b}$ is the mean SBH and $\sigma_{s}$ is the standard deviation of the barrier height distribution. This model also leads to a voltage dependence of $\phi_{b}$ given by:

$-\frac{\partial \phi_{b}}{\partial V}=\frac{1}{n}-1=-\rho_{2}+\rho_{3} \frac{q}{2 k T}$

where $\rho_{2}$ and $\rho_{3}$ correspond respectively to the voltage coefficient dependencies of the mean SBH $\phi_{b}$ and its standard deviation $\sigma_{s}^{2}$ when the ideality factor $n$ is voltage independent. Data of Fig. 8 have been analyzed using Eqs. (11) and (12) for each of the two temperature ranges. Table 2 summarizes the values of $\bar{\phi}_{b}, \sigma_{s}, \rho_{2}$ and $\rho_{3}$ derived from this analysis. Two Gaussian distributions, with mean barrier heights equal to 0.76 and 1.03 and corresponding standard deviations

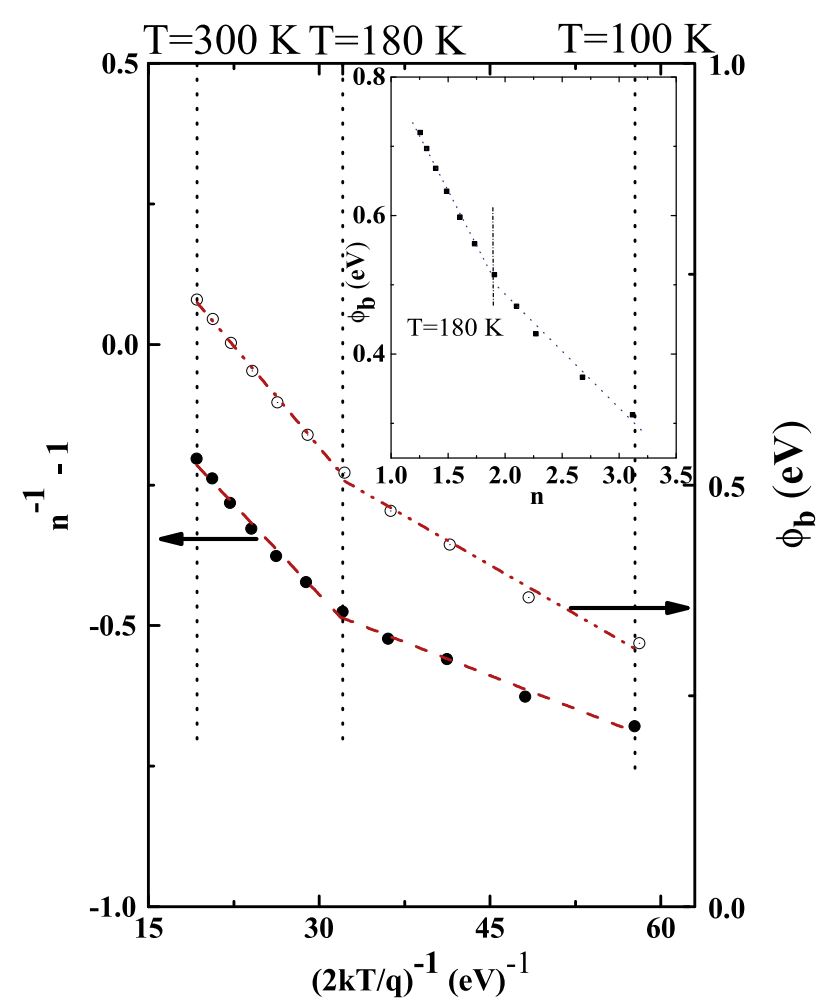

Fig. 8. Barrier height $\phi_{b}$ and $\left(n^{-1}-1\right)$ versus $\frac{1}{2 k T}$ in the Pt/n-GaN Schottky diodes. The inset shows the barrier height $\phi_{b}$ as a function of $\mathrm{n}$ for the structure under test. 
of 89 and 127 for low and high temperature range, respectively, are needed to fit experimental results.

Accordingly, the voltage variations of $\bar{\phi}_{b}$ and $\sigma_{s}^{2}$ can be written [27] as:

$\bar{\phi}_{b}(V, T)=\bar{\phi}_{b}(0, T)+\rho_{2} V$

and

$\sigma_{s}^{2}(V)=\sigma_{s}^{2}(0)+\rho_{3} V$.

As shown in Table 2 the values of $\rho_{3}$ are negative for both temperature ranges, indicating a decrease of $\sigma_{s}^{2}$ and thus a more homogeneous SBH with increasing voltage. By contrast, the mean SBH exhibits a positive value of the voltage coefficient for low temperature and a negative value for high temperature. The negative value of $\rho_{2}$ corresponds to a decrease of $\bar{\phi}_{b}$ with increasing voltage that can be explained by the image force lowering of the barrier height. In the low temperature range, the tunneling current is preponderant yielding, a limitation of the image force effect and the $\bar{\phi}_{b}$ increases with the voltage. Finally, according to Eqs. (11) and (12), the voltage dependence of the apparent SBH $\phi_{b}$ can be written as:

$\phi_{b}(V, T)=\bar{\phi}_{b}(0, T)-\frac{q}{2 k T} \sigma_{s}^{2}(0 V)+\left(\rho_{2}-\frac{q \rho_{3}}{2 k T}\right) \times V$

indicating an increase of the apparent SBH with increasing voltage whatever the sign of the coefficients $\rho_{2}$ and $\rho_{3}$ given in Table 2 .

The inset of Fig. 8 shows the dependence of $\phi_{b}$ with $n$; again this dependence is linear, but with different slopes, in both low and high temperature ranges. This is a clear indication of a laterally inhomogeneous distribution of barrier heights as reported by Schmitsdorf et al. [38]. From the data of the inset of Fig. 8 (extrapolated to $n=1$ ), we can derive the value of the real barrier height which is equal to $0.794 \mathrm{eV}$ for temperatures above $180 \mathrm{~K}$, in a rather good agreement with the values obtained at room temperature.

Therefore, all these results show that, in accordance with Roccaforte et al. [39], the distribution of the interface state density can be pointed out as responsible for the temperature dependence of $\phi_{b}$ and $n$. The interface state density is linked to the high dislocation density, generally observed in the GaN near surface region, which acts as defect traps, leading to a high interface defects density. The latter can partially pin the Fermi level, resulting in the wide variability of the Schottky barrier height values found in the literature, which are generally lower than the theoretically predicted ones. These interface states can also affect the value of the surface potential and thus induce some mistakes in the determination of the value of the barrier height via C-V measurements, as we will show subsequently. As seen in the previous section, in Fig. 2 the intercept of the $1 / C^{2}$ plot with the voltage axis gives the built in voltage $V_{b i}$ which is used to calculate the flat band barrier height $\phi_{b F b}$ by means of the relationship:

$\phi_{b F b}=V_{b i}+\frac{k T}{q} \ln \frac{N_{c}}{N_{D}}+\frac{k T}{q}$

where $N_{c}$ is the effective density of states in the conduction band. We have then determined the flat band barrier height $\phi_{b F b}$ and reported

Table 2

Mean SBH, standard deviations and their voltage dependencies coefficient as determined from the barrier inhomogeneities at Schottky contact model [27].

\begin{tabular}{lcc}
\hline & Below $180 \mathrm{~K}$ & Above $180 \mathrm{~K}$ \\
\hline$\phi_{b}(\mathrm{eV})$ & 0.76 & 1.03 \\
$\sigma_{s}(\mathrm{meV})$ & 89 & 127 \\
$\rho_{2}$ & 0.233 & -0.197 \\
$\rho_{3}(\mathrm{eV})$ & -0.008 & -0.0214
\end{tabular}

the results in Fig. 4-b. The values of the barrier height $\phi_{b F b}$ deduced from $\mathrm{C}-\mathrm{V}$ measurements are significantly different and larger than those deduced from I-V measurements (Fig. 4-a) and do not decrease with increasing temperature. These values must be corrected to take into account the non-negligible values of $n$ and $R_{s}$. The correction consists of the calculation of $\phi_{b F b}$ using the real built-in voltage $V_{b i c}$ [25], which does not correspond to the applied voltage due to a screening effect of the interface states, given by:

$V_{b i c}=\frac{V_{b i}-R_{s} \times I_{0}}{n}$,

where $I_{o}$ is the current measured at the voltage $V=V_{b i}$. Finally, once the corrections are made, we have obtained the values reported in Fig. 4-c. The temperature dependence of $\phi_{b}$ as deduced from I-V (Fig. 4-a) and C-V (Fig. 4-c) measurements is in very good agreement and exhibits similar trends. The values of the two barrier heights $\phi_{b}$ and $\phi_{b F b}$ are also in rather good agreement, confirming our approach. The initially observed discordance between $\phi_{b}$ and $\phi_{b F b}$ is clearly due to the fact that the $\frac{1}{c^{2}}-V$ curve must be plotted against the real surface potential in the semiconductor, which is very different from the applied voltage as considered for ideal Schottky contacts.

\section{Conclusion}

I-V and C-V characterizations of Pt/n-GaN Schottky diodes have been performed as a function of temperature and analyzed in the framework of thermionic and thermionic field emissions. The results show an increase of the Schottky barrier height $\phi_{b}$ and a decrease of the ideality factor $n$ both with the increase of the temperature. We have shown that this behavior originates in the existence of an interface state density distribution, which is determined via the analysis of the temperature dependence of the I-V measurements, and allows the tunneling of the carriers from the semiconductor to the metal. The broad peak in the energy dependence of the interface state distribution centered at an energy equal to $0.5 \mathrm{eV}$ has been attributed to nitrogen anti-sites and confirmed by DLTS results reported in the literature. These interface states have been shown to be also responsible for the low apparent barrier height and higher-than-one ideality factors obtained at low temperature.

The deduced mean SBH values are equal to $1.03 \mathrm{eV}$ and $0.76 \mathrm{eV}$ respectively for the high and low temperature ranges. The first value could be rather related to the metal/semiconductor contact as its value is close to the literature values found for Pt/nGaN Schottky contacts (see Table 1). The second value reveals the effect of the interface states responsible for such decrease of the SBH, and mainly visible at low temperature. The standard deviation decreases with increasing voltage whatever the temperature range, indicating a narrowing of the barrier distribution and consequently a more homogenous interface. The interface states are also responsible for the small modulation of the surface potential inside the semiconductor, as revealed by the analysis of the $\mathrm{C}-\mathrm{V}$ measurements, which without correction of the built in voltage, can lead to erroneous values of the Schottky barrier height $\phi_{b}$. The development of high quality Schottky diodes on GaN is still closely related to the passivation of these interface states.

\section{References}

[1] J.S. Foresi, T.D. Moustakas, Appl. Phys. Lett. 62 (1993) 2859.

[2] J.D. Guo, M.S. Feng, R.J. Guo, F.M. Pan, C.Y. Chang, Appl. Phys. Lett. 67 (1995) 2657.

[3] L. Wang, M.I. Nathan, T.-H. Lim, M.A. Khan, Q. Chen, Appl. Phys. Lett. 68 (1996) 1267

[4] T.U. Kampen, W. Mönch, Appl. Surf. Sci. 117 (118) (1997) 388.

[5] S.N. Mohammad, H. Morkoç, Prog. Quantum Electron. 20 (1996) 361.

[6] S.J. Pearton, J. Zolper, R.J. Shul, F. Ren, J. Appl. Phys. 86 (1999) 1.

[7] D. Théron, C. Gaquière, J.D. Jaeger, S.L. Delage, in: 12th GaAs Symposium-Amsterdam, 2004, p. 547.

[8] S. Petroni, G. Tripoli, C. Combi, B. Vigna, M.D. Vittorio, M.T. Todaro, G. Epifani, R. Cingolani, A. Passaseo, Superlattices Microstruct. 36 (2004) 825.

[9] P. Hacke, T. Detchprohm, K. Hiramatsu, N. Sawaki, Appl. Phys. Lett. 63 (1993) 2676. 
[10] A. Schmitz, A.T. Ping, M.A. Khan, Q. Chen, J.W. Yang, I. Adesida, Semicond. Sci. Technol. 11 (1996) 1464

[11] Y. Kribes, I. Harrison, T.S. Cheng, C.T. Foxon, Part. Sci. Technol. 12 (1997) 913.

[12] L. Yu, Q. Liu, J. Xing, D. Qiao, S.S. Lau, J. Appl. Phys. 84 (1998) 2099.

[13] M. Jang, J. Lee, ETRI J. 24 (2002) 455.

[14] S. Oyama, T. Hashizume, H. Hasegawa, Appl. Surf. Sci. 19 (2002) 323.

[15] X.M.S.Y. Sun, J. Wang, D.G. Zhao, G. Feng, Y. Fu, S.M. Zhang, Z.H. Zhang, Z.H. Feng, Y.X. Bai, H. Yang, J. Phys. D 35 (2002) 2648

[16] B. Akkal, Z. Benamara, H. Abid, A. Talbi, B. Gruzza, Mater. Chem. Phys. 85 (2004) 27.

[17] J.S. Jang, D. Kim, T.Y. Seong, J. Appl. Phys. 99 (2006) 73704:1

18] M. Mamor, J. Phys. Condens. Matter 21 (335802) (2009) 1.

[19] W. Mtangi, P.J.J. van Rensburg, M. Diale, F.D. Auret, C. Nyamhere, A.C.J.M. Nel, Mater. Sci. Eng. B 171 (2010) 1.

[20] N. Yildirim, K. Ejderha, A. Turut, J. Appl. Phys. 108 (2010) 114506.

[21] H. Kim, S.N. Lee, Y. Park, J.S. Kwak, T.Y. Seong, Appl. Phys. Lett. 93 (2008) 032105

[22] E.H. Rhoderick, R. Williams, Metal Semiconductor Contact, Clarendon Press, Oxford, 1988.

23] S.M. Sze, Physics of Semiconductor Devices, 2nd edition Wiley Interscience, 1981.

[24] S. Aydoğan, K. Çinar, H. Asil, C. Coşkun, A. Türüt, J. Alloys Compd. 476 (2009) 913.
25] A. Ahaitouf, A.Z. Ahaitouf, J.P. Salvestrini, H. Srour, J. Semicond. 32 (2011) 104002:1.

[26] S.K. Cheung, N.W. Cheung, Appl. Phys. Lett. 49 (1986) 85.

[27] H. Werner, H. Güttler, J. Appl. Phys. 69 (1991) 1522.

[28] R. Tung, Phys. Rev. B 45 (1992) 13509.

[29] J.D. Levine, J. Appl. Phys. 42 (1971) 3991

[30] C. Crowell, Solid State Electron. 20 (1977) 171.

[31] H.C. Card, E.H. Rhoderick, J. Phys. D 4 (1971) 1589.

[32] K.S.K. Maeda, H. Ikoma, T. Ischida, Appl. Phys. Lett. 62 (1993) 2560.

[33] A. Ahaitouf, A. Bath, E. Losson, E. Abarkan, Mater. Sci. Eng. B 52 (1998) 208

[34] S.J. Pearton, C.R. Abernathy, F. Ren, Gallium Nitride Processing for Electronics Sensors and Spintronics, Springer-Verlag, London, 2006.

[35] H.K. Cho, K.S. Kim, C.H. Hong, H.J. Lee, J. Cryst. Growth 223 (2001) 38.

[36] W. Götz, N.M. Johnson, Appl. Phys. Lett. 65 (1994) 463.

[37] T. Tsarova, T. Wosinński, A. Macosa, C. Skiierbiszewski, I. Grzegory, P. Perlin, Acta Phys. Pol. A 112 (2007) 331.

[38] R.F. Schmitsdorf, T.U. Kampen, W. Mönch, J. Vac. Sci. Technol. B 15 (1997) 1221

[39] F. Roccaforte, F. Giannazzo, F. Iucolano, J. Eriksson, M.H. Weng, V. Raineri, Appl. Surf. Sci. 256 (2010) 5727. 